\title{
Créativité et incertitude dans les nouveaux rituels contemporains
}

\author{
Anna Fedele
}

Résumé : Cet article se fonde sur une enquête de terrain menée auprès de Portugais, d'Italiens, de Catalanes et d'Espagnoles, en grande majorité des femmes, influencés par le mouvement transnational de la spiritualité de la Déesse. En s'appuyant sur les récits de ces pratiquantes, il analyse le rôle du doute et de l'incertitude dans les rituels créés dans le cadre de cette spiritualité. L'auteure montre que ces nouvelles pratiques contemporaines donnent un accès privilégié à l'incertitude inhérente au rituel, les participantes parlant plus librement de leurs doutes et des stratégies qu'elles emploient pour les dissiper que s'il s'agissait de cérémonies consacrées par une tradition séculaire. En m'appuyant sur les travaux de Simon Coleman $(2002,2009$, 2013) sur le pèlerinage et le rituel, je suggère que l'incertitude joue un rôle non seulement dans les rituels de la spiritualité new age, mais également dans les autres rituels contemporains, religieux ou profanes, élaborés dans un contexte occidental, pluriel et de plus en plus sécularisé.

Mots clés : certitude, spiritualité new age, créativité rituelle, spiritualité de la Déesse, Europe du Sud, incertitude.

\begin{abstract}
This paper is based on fieldwork among Portuguese, Italians, Catalans and Spaniards influenced by the transnational Goddess spirituality movement. Through an analysis of ritual narratives I will analyze the role of doubt and uncertainty in contemporary rituals created within Goddess spirituality. I will show that contemporary crafted rituals offer a privileged window upon the uncertainty intrinsic in ritual because participants feel less constrained by a long lasting religious tradition and talk more openly about their doubts and their strategies to neutralize them. Drawing on Simon Coleman's analysis of pilgrimage and ritual (2002, 2009, 2013) I suggest that uncertainty may play an important role not only in rituals created in the context of New Age spirituality but also in other contemporary rituals created in plural and increasingly secularized Western contexts.
\end{abstract}

Keywords: certainty, New Age spirituality, crafted rituals, Goddess spirituality, Southern Europe, uncertainty. 
Ce texte analyse le rôle du doute et de l'incertitude dans des rituels contemporains, qui ont été créés dans le cadre de la spiritualité de la Déesse ${ }^{1}$. Il se fonde sur une recherche consacrée à la diffusion de ce mouvement spirituel dans les pays de tradition catholique de l'Europe du Sud. Issues de familles catholiques du Portugal, d'Italie, de Catalogne espagnole ou d'Espagne, les personnes que j'ai rencontrées en travaillant sur le terrain critiquent le christianisme, jugé misogyne et patriarcal, tout en continuant de vénérer des figures chrétiennes telles que la Vierge Marie, Jésus ou Marie Madeleine. En adaptant de façon créative des théories et des pratiques néo païennes en provenance des États-Unis et de la Grande-Bretagne, ces personnes rendent un culte à des divinités féminines - la « Déesse », la «Terre Mère »-, mais ne se décrivent pas eux-mêmes comme des «sorciers» ou des «païens » (Fedele 2013a, 2013b). Grâce à ces rituels, elles tentent de combiner des figures, des symboles et des gestes païens avec ceux du christianisme.

D'après les théories classiques des sciences humaines, les formes multiples du religieux constituent un remède contre l'incertitude. Même si le rituel a fait l'objet d'approches diverses (par exemple, Geertz 1973, Bell 1992, 1997, Rappaport 1999, Handelman et Lindquist 2004), la recherche privilégie généralement sa capacité de susciter chez ses participants un sentiment de certitude et une impression de continuité (Coleman 2013). Le pouvoir explicatif de ces approches est incontestable, mais je me place ici dans une perspective légèrement différente. L'incertitude inhérente au rituel a reçu peu d'attention jusqu'à présent. Ce type d'incertitude peut venir des participants qui doutent de sa réussite, de l'officiant qui cherche à organiser au mieux son déroulement, ou encore d'événements incontrôlables qui gênent, voire interrompent le processus de ritualisation.

Or, les adeptes de la spiritualité de la Déesse ont bien conscience de créer leurs rituels et d'en tester les effets. À l'instar de Catherine Bell dans son analyse des nouveaux rituels contemporains, il m'est apparu que l'un des traits principaux de ce type de rituels consiste à « montrer que ça marche » (1997:241). Dans ce contexte, l'officiant n'est plus une autorité incontestable et peut faire l'objet de critiques. Ne subissant pas le poids d'une tradition religieuse séculaire, les participants parlent plus ouvertement de leurs doutes et des stratégies qu'ils adoptent pour les dissiper, si bien que ces rituels nous offrent un aperçu privilégié des processus de l'incertitude propre à ces pratiques. En m'inspirant

\footnotetext{
${ }^{1}$ Une première version en anglais de cet article a été présentée à la table ronde, « Dealing with Doubts, Putting to Test : the Importance of Uncertainty in Vernacular Religion », que j'ai coordonnée avec Élisabeth Claverie à la 12 ème conférence biannuelle de 1'Association Européenne des Anthropologues Sociaux, organisée à Nanterre en 2012. Pour leurs commentaires et leurs suggestions, je souhaite remercier Élisabeth Claverie et les participants : Monique Scheer, Agnès Clerc-Renaud, Emma Aubin Boltanski, Stanley Brandes, Anne-Marie Losonczy, Silvia Mesturini-Cappo, Kim Knibbe, Alessandra Brivio, Raquel Romberg et Peter Collins. Les remarques d'Ellen Badone notamment ont constamment enrichi cette table ronde. Je remercie également William Christian, Antoinette Molinié et Michael Houseman pour leurs critiques constructives, ainsi que Sylvie Muller pour son aide avec la version française de cet article, sa lecture attentive et ses suggestions. Enfin, merci à Simon Coleman, qui m'a autorisée à utiliser avant sa parution son dernier article consacré au rituel et au pèlerinage (2013).
} 
de l'analyse du pèlerinage et du rituel de Simon Coleman (2009, 2013, et Elsner 1998), je montre également qu'il est important de tenir compte du rôle joué par cette incertitude rituelle dans un contexte occidental, pluriel et de plus en plus sécularisé.

\section{Création de rituels menstruels à la Sainte Baume}

Depuis les années 1990, un nombre croissant de personnes en Occident déclarent qu'elles ne sont «pas religieuses mais spirituelles» (par exemple, Pike 2004 ou Fedele et Knibbe 2013) ${ }^{2}$. La religion est en effet associée à la hiérarchisation, à la domination masculine et à la condamnation du corps et de la sexualité. Pour les pratiquants spirituels, une force de vie ou « énergie » imprègne toute chose ; le divin habite le monde, lui est immanent, et se trouve aussi dans chaque être humain. Mes interlocutrices accusent souvent les religions monothéistes d'être les principales responsables de l'exploitation de la planète et de l'oppression des femmes, parce que tout en se fondant sur un dieu masculin et transcendant, elles font de la terre un monde souillé par le péché.

L'utilisation du genre comme outil de critique religieuse et sociale (Eller 1993:6 ; Fedele 2013a : 67) est particulièrement évidente dans la spiritualité de la Déesse. En se réappropriant un passé matriarcal plus ou moins fantasmé (Eller 2000), mes interlocutrices souhaitent raviver les religions pré chrétiennes de nos ancêtres européens (Fedele 2013a: 10-11, 68). Je me limite ici à l'analyse d'un seul rituel, organisé à la Sainte-Baume dans le sud de la France, qui fait partie du «pèlerinage initiatique du sang », l'une des principales activités du groupe du Bois de la Déesse, avec lequel je suis en contact depuis 2004. Ce rituel illustre bien la façon dont ce groupe, à l'instar d'autres que j'ai rencontrés en Europe du Sud, créent leurs propres rituels en puisant dans la gestuelle de traditions dites «indigènes », rituels dont ils expliquent l'efficacité en recourant à une terminologie scientifique et aux théories de la psychologie et de l'anthropologie.

Créé en 2001 par Dana, une Argentine approchant la cinquantaine, le Bois de la Déesse compte, en 2004, jusqu'à trois cents femmes, réparties dans presque toute l'Espagne. Un noyau dur d'une bonne trentaine de personnes participe de façon régulière aux rituels ou aux ateliers organisés par Dana, le reste du groupe y venant de façon plus occasionnelle. La plupart des membres ont entre 35 et 55 ans, même si l'âge varie de 24 à 65 ans. Originaires des couches moyenne et inférieure de la classe moyenne, elles occupent des emplois très divers, certaines exerçant une seconde activité comme

\footnotetext{
${ }^{2}$ Les personnes qui se qualifient elles-mêmes de «spirituelles » sont parfois rangées dans la catégorie «new age ». La plupart de celles que j'ai rencontrées jugeant ce terme méprisant, j'évite de l'utiliser (Fedele 2013 : 277 n. 2). Les problèmes que pose cette expression sont examinés en détail par Wood(2007); voir également Fedele et Knibbe (2013).
} 
thérapeute en médecine alternative (Fedele 2013a). Aujourd'hui, le Bois de la Déesse est devenu l'un des groupes de la spiritualité de la Déesse les plus grands d'Espagne.

En 2004, en tant qu'observatrice participante, j'ai accompagné vingt-huit de ces femmes en Provence, dans un pèlerinage de six jours, qui incluait la visite des Saintes-Maries-de-la-Mer, de Marseille et de la Sainte-Baume. Ce «pèlerinage initiatique du sang », qui fait suite au « chemin de Marie Madeleine $»^{3}$, comporte une offrande collective de sang menstruel, présentée à la « Terre Mère » sur le massif de la Sainte-Baume, à une trentaine de kilomètres de Marseille. Même si elles reprochent au christianisme, au catholicisme en particulier (ou à «l'Église» comme elles les désignent en général l'un et l'autre), son androcentrisme et la persécution des sorcières, les membres $\mathrm{du}$ Bois de la Déesse croient néanmoins au pouvoir de l' « enseignement mystique chrétien $»^{4}$. Préservé par des groupes hérétiques comme les cathares ou les Templiers », cet enseignement porterait sur les pouvoirs de connaissance et de guérison dont certains lieux saints sont encore investis. Ces femmes trouvent la matière première nécessaire à l'élaboration de leurs propres théories et rituels dans une sorte de littérature " ésotérico-spirituelle » : un corpus de textes mêlant anthropologie, psychologie, histoire et science des religions (Fedele 2006, 2013a). «Inconscient collectif », « archétypes », « rituels initiatiques », « rites de passage », mais aussi « ADN », « théorie quantique » ou « champ morphique », ce type de vocabulaire abonde dans le discours des praticiennes spirituelles.

Selon le Bois de la Déesse, les «femmes amérindiennes » (Native American women) qui avaient leurs règles toutes en même temps, leur cycle de 28 jours environ s'accordant avec celui de la lune, se réunissaient dans des huttes menstruelles (moon huts) où, assises à même le sol moussu, elles laissaient leur sang s'écouler directement dans la Terre. En offrant elles aussi leur sang à la « Terre Mère », les membres de ce groupe font ainsi renaître (revitalize) un rituel qui aurait existé dans une ancienne société matriarcale, avant d'être balayé par le tabou judéo-chrétien de la menstruation. L'imposition progressive de valeurs patriarcales aurait rendu impur et même dangereux le flux qui marque la période de fertilité de la femme, et lui donne le pouvoir d'enfanter (Fedele 2014).

Le soir de leur arrivée à la Sainte-Baume, les pèlerines se rassemblent dans la salle de réunion du foyer catholique qui les héberge, afin de préparer leur offrande de sang menstruel à la Terre Mère. Pour cette cérémonie, Dana se fait assister d'une «experte rituelle», Clara, et d'une « experte scientifique », Puri, qui est médecin. Les participantes forment un cercle autour d'un « autel » placé au centre de la pièce. Elles ont apporté leur « équipement de prêtresse » (priestess kit) qui comprend un

\footnotetext{
3 Pour ce pèlerinage et le lien entre Marie Madeleine et la Sainte Baume, voir Fedele 2013a, donne également une description plus détaillé du pèlerinage du sang.

${ }^{4}$ La plupart des croyances de ces pèlerines se retrouvent dans le mouvement nord-américain de spiritualité néopaïenne et féministe décrit, entre autres, par Cynthia Eller (1993) et Sarah M. Pike (2001, 2004).
} 
morceau de coton imbibé de sang menstruel séché. Les femmes ménopausées, et celles qui ont oublié d'apporter leur prélèvement offriront une goutte de sang, qui sera prélevé par Puri en les pi quant au doigt avec une lancette servant à mesurer la glycémie.

L'experte scientifique nous explique que le sang de toutes les femmes sera mélangé à l'intérieur d'un calice dans une solution aqueuse alcoolisée, afin d'obtenir une «teinture mère » commune (unified). Selon des principes inspirés de l'homéopathie, une seule goutte de cette teinture sera diluée dans un flacon de $30 \mathrm{ml}$ rempli d'eau et d'alcool. Outre d'éviter une contamination accidentelle, cette dilution permettra d'augmenter le «pouvoir» de la teinture de départ et, en modifiant les «informations» contenues dans l'ADN de chacune des femmes, de les rendre «plus subtiles $\gg$.

Le jour suivant, chaque participante devra secouer la bouteille pendant un certain le temps, tout en y « transférant» ce qu'elle veut changer ou « lâcher» dans sa vie. Ce processus favorisera la création d'une «âme de groupe », et la potion obtenue aidera chacune à guérir ses blessures et à modifier des comportements dangereux ou autodestructeurs.

Ce mélange créatif d'homéopathie et de génétique me laisse perplexe, et je ne suis pas la seule dans ce cas. L'air plutôt perdu, les participantes émettent des doutes. Dana reconnaît qu'elle s'est d'abord sentie un peu dépassée elle aussi par cette approche fondée sur l'ADN, mais Puri «a mené des recherches sur le sujet » et fait l'expérience de son pouvoir; en outre, de plus en plus de personnes s'en servent dans le monde entier. De toute façon, ajoute-t-elle, le plus important c'est que le rituel va créer une relation de sororité par le sang, tout en reliant les participantes aux femmes du précédent pèlerinage organisé deux ans auparavant. Ce n'est pas la première fois que, dans une situation critique, face à un groupe désorienté, Clara prend le contrôle pour, à sa façon simple et directe, ramener tout le monde les pieds sur terre : comme les femmes autrefois, qui labouraient les champs en laissant leur flux s'écouler dans le sillon qu'elles venaient d'ouvrir, les pèlerines offriraient leur sang à la « Terre Mère ").

Faute de place pour décrire en détail le reste du rituel, disons simplement que chaque femme a purifié et béni son obole de sang en le faisant passer dans la fumée du copal, une résine aromatique utilisée par les Amérindiens, puis s'est avancée à genoux vers le calice pour 1'y déposer'5.

L'offrande collective à la Terre se passe la nuit suivante dans le bois de la Sainte-Baume. Au cours d'une cérémonie élaborée, les femmes sont purifiées par Clara avec le copal avant de prendre place, chacune selon son âge, dans un cercle rituel; puis l'une après l'autre elles boivent dans le calice, où quelques gouttes de la dilution sanguine ont été ajoutées à un vin de Provence. J'ai montré

\footnotetext{
${ }^{5}$ Ce rituel est décrit et analysé en détail dans Fedele $2013: 145-190$.
} 
ailleurs qu'en reproduisant l'Eucharistie, cette pratique propose une inversion rituelle de la conception chrétienne de Dieu et du tabou menstruel (2013 : 169-186, 2014).

\section{Récits rituels et incertitude}

J'utilise ici le mot « rituel» au sens large pour désigner des pratiques considérées par les personnes mêmes qui les élaborent comme des rites nouveaux ou renouvelés, destinés à établir un contact avec des êtres méta-empiriques et à obtenir la capacité de discerner, d'agir et de guérir.

$\mathrm{Ni}$ répétitifs ni stéréotypés, ces rituels, nous l'avons vu, sont créés de manière ad hoc en fonction de la situation. Pour moi, qui m'inspire de l'étude d'Élisabeth Claverie sur les pèlerins français se rendant à Medjugorje en Bosnie-Herzégovine (2003), les acteurs sociaux ne sont pas les victimes passives ni du milieu où ils ont grandi ni des théories associées à la spiritualité qu'ils ont embrassée. Tout pratiquant est à même d'affronter la réalité et d'exercer son sens critique : avant d'adhérer à une théorie ou d'accepter de nouvelles règles, il les met en question. Aussi me suis-je particulièrement intéressée, au cours de ma recherche, à la réception que les pèlerines réservaient à certaines théories et aux processus qui les conduisaient à les accepter ou à les rejeter, afin d'établir leur sélection personnelle de théories, de comportements et d'actions rituelles.

Le discours autour d'un rituel est le produit d'un processus complexe d'interprétation. L'intention des célébrants, leur expérience et leur analyse du rituel qui leur est proposé par un chef ou leader, nous permet de comprendre et les modifications qu'ils lui apportent et son fonctionnement. Selon Ronald Grimes, « il n'existe aucune bonne raison d'exclure les récits rituels, en particulier lorsque les participants eux-mêmes se servent d'histoires autobiographiques pour donner un sens à un rite. [...] Si les gens racontent des histoires personnelles sur des moments de passages, alors ces témoignages participent en toute légitimité de la signification du rite » (2000: 10). De plus, c'est principalement grâce à ces récits rituels que nous pouvons entrevoir l'incertitude à laquelle les participantes font face. Et c'est grâce à eux également que nous pouvons estimer le degré d'implication de l'une ou l'autre d'entre elles, et les stratégies mises en œuvre pour tester l'efficacité d'un rituel ou pour dissiper les doutes qui l'empêchent de profiter pleinement de la cérémonie, comme dans le cas de Maria Antònia ci-dessous.

Au moment du pèlerinage de l'été 2004, Maria Antònia, une pèlerine de Catalogne espagnole au début de la cinquantaine, vient d'entrer dans la ménopause. Ses dernières règles remontant au mois de juillet 2003, elle a accepté ce changement et ne s'inquiète pas de n'avoir pu apporter un échantillon de son sang menstruel. Elle s'est sentie à l'aise pendant le rituel de l'ADN et, m'a-t-elle confié, il lui a semblé qu'elle savait d'avance ce qu'elle devait faire, comme si cela faisait déjà 
partie d'elle. Au cours du rituel célébré dans le bois, elle a toutefois éprouvé quelques difficultés, du moins au début :

Quand le moment est venu de faire l'offrande personnelle, j'étais un peu nerveuse. Je voyais les autres femmes qui allaient vers le centre devant moi, et je me suis mise à me demander si je serai capable de faire le rituel. [...] J'ai eu peur de ne pas être capable de faire ce qu'on attendait de moi.

J'ai passé un moment difficile, comme si je m'étais déconnectée de cette explosion intérieure que j'avais ressentie auparavant. [...] Mais au moment même où j'y suis allée, toute mon anxiété a disparu. [...] À nouveau j'ai ressenti la même chose que la nuit précédente : je savais exactement ce que je devais faire et dire. Je n'avais aucun doute; autrement dit, je me sentais à nouveau comme la Déesse (Le 4 juin 2005, Fedele 2013a :172-173).

Il a ainsi fallu un moment à Maria Antònia, qui s'est d'abord sentie incompétente, pour apprécier ce rituel. D'autres pèlerines en ont également critiqué la complexité, soulignant à quel point il était difficile d'y entrer pleinement. N'ayant reçu que des explications très générales sur la façon de procéder, plusieurs d'entre elles ne savaient pas comment elles étaient censées se comporter. Sol, une squatter au début de la trentaine à laquelle je fais part de mes doutes, me dit: «Peu importe, une graine a été semée ». Elle m'explique que l'exactitude des gestes compte moins que l'exécution du rituel, lequel agirait comme un germe se développant lentement.

Je n'ai pas tardé à découvrir qu'il existait autant de versions du rituel célébré dans le bois (et de sa bonne exécution) qu'il y avait de participantes. Comme j'ai pu l'observer en d'autres occasions, ce qui importe pour les pratiquants spirituels ce n'est pas la précision des gestes ni l'exactitude des paroles prononcées, mais les symboles (le calice, le cercle des femmes, la bougie), les actes symboliques (boire au calice, prendre la parole, se tenir au centre du cercle) et leurs effets (voir Houseman 2007, 2010). L'efficacité du rituel est loin d'aller de soi pour les pèlerines, qui abordent le processus de ritualisation d'un œil critique, confrontant ses effets supposés à leurs « perceptions énergétiques » pendant et après l'offrande.

D'autres participantes se sont également senties mal à l'aise et incompétentes au cours de la cérémonie. Felicia, la cinquante, membre du noyau dur, qui a aimé le rituel de l'ADN, est très déçue de n'avoir pas réussi à se mettre en phase avec le rituel du bois. La façon dont les rituels sont accomplis est souvent pour elle, avoue-t-elle, source d'inquiétude. Felicia fait partie des membres les plus âgées et les plus engagées du Bois de la Déesse et, à ce titre, elle se sent responsable du bien-être des pèlerines et de la bonne exécution du rituel ; ce qui lui donne une perspective tout à 
fait différente. Deuxième à entrer dans le cercle, elle ne tarde pas à réaliser que la plupart des participantes grelottent de froid, assises pieds nus à même la terre. La majorité d'entre elles n'ont pas l'habitude de rester immobiles si longtemps sans pouvoir s'adosser. Pour Felicia, le scénario du rituel n'a pas été respecté et, à la différence de Maria Antònia, elle n'a pu dissiper ses doutes.

Si le rituel comporte des gestes, j'aime bien qu'ils soient faits avec la plus grande précision possible. Mais quand on a invoqué les sept directions de l'espace, il y a des personnes qui le faisaient bien et d'autres qui m'ont semblé ne pas le faire correctement. Et j'étais mal. Ce n'était pas tant que l'invocation puisse être mal faite qui me posait problème, mais plutôt que c'est tellement plus beau quand on la fait en se sentant à l'aise, et c'est mieux pour le cercle si on voit de beaux gestes au lieu d'une personne qui est mal, parce qu'elle ne sait pas si elle est censée les faire comme ci ou comme ça (Le 7 juin 2005, Fedele 2013a :174).

Pour Ruth également, placée juste après moi dans le cercle pendant l'offrande, les doutes et les questions sont une source potentielle de distraction. Quand je lui demande de parler de son expérience du pèlerinage, elle me répond qu'elle ne se sent pas spécialement liée à Marie Madeleine et que je ferais sans doute mieux de m'adresser à quelqu'un d'autre. Elle s'est jointe au pèlerinage « pour voir ce que c'était et pour passer du temps avec son amie Carme », et non pas pour répondre à « un appel spirituel ». Le point de vue de Ruth me paraît intéressant parce qu'elle représente une sorte de «pèlerin limite » qui, motivé par la curiosité, n’a ni attente forte ni but particulier. Elle constitue un bon exemple du «semi-engagement rituel» (ritual semi-engagement) (2013) que Coleman qualifie de « participation latérale » (lateral participation) (2009, 2013).

Dès le début du pèlerinage, Ruth a eu le sentiment que si elle prenait le rituel et ses implications dans un sens trop littéral, elle n'en tirerait aucun bénéfice. À l'instar d'autres pèlerines dans la même situation, elle a décidé de ne pas accorder trop d'importance aux explications de Dana et de Puri, et de fixer son attention sur un niveau plus abstrait ou « symbolique ». Car conclut-elle, le plus important comme l'a dit Clara c'est que des femmes célèbrent ensemble un rituel.

Chaque fois que je voyais la petite bouteille [avec le sang dilué], je me disais " mais qu'est-ce qu'on est en train de faire?» [Elle rit.] [...] Et l'offrande, je n'ai pas saisi ça non plus [...] l'offrande de mon propre sang, je me suis demandée: "Comment une goutte de sang aussi insignifiante peut-elle jouer un rôle dans quelque chose d'aussi important?" Mais en réalité, c'était comme si on rendait grâce pour ce qu'on vivait ensemble. Si tu la vois à ce niveau, l'offrande, tu peux à la rigueur lui trouver un sens, mais si tu restes sur un niveau normal tu es 
perdue, et tu commences à te dire "mais qu'est-ce que je fous et qu'est-ce qui se passe » (May 16, 2005, Fedele 2013a : 175).

D'après les remarques de Ruth, c'est en mettant l'accent sur le corps genré et sur la formation d'un groupe que les pèlerines dissipent leurs doutes. Ruth ne peut croire au pouvoir de son sang et préfère, comme Felicia, se concentrer sur l'importance du groupe, et sur le fait que ce saignement est ce qu'elle partage avec toutes les autres femmes.

À notre retour à l'auberge, affamées, il est minuit passé. Tout en se préparant un repas puis en le mangeant dans la cuisine commune, les pèlerines parlent avec animation de ce qu'elles ont vécu et ressenti pendant le rituel. Malgré les difficultés et les moments de perplexité, elles semblent éprouver un sentiment nouveau de communion, et ne cessent de répéter : «Maintenant, nous sommes des sœurs de sang !».

Après le repas, à l'invitation de Dana, les participantes tiennent une brève réunion dans la salle commune avant d'aller dormir. Soudain, une retardataire entre dans la pièce avec son chien, le « seul membre mâle du groupe » et la mascotte du pèlerinage. Il vient de manger un tampon usagé qu'il a trouvé dans la salle de bain, nous apprend-t-elle : « il voulait lui aussi participer au rituel de partage du sang !» Naturellement, le comportement du chien est interprété comme une preuve supplémentaire du pouvoir du rituel de la cérémonie qu'elles viennent de vivre.

\section{Analyse du rituel}

Depuis une vingtaine d'années, les pratiques rituelles ont gagné en visibilité et en importance dans des contextes aussi bien religieux que laïques (notamment, Aune et de Marinis 1996; Grimes 2000 ; Fellous 2001 ; Dianteill et al. 2004 ; Grimes et al. 2011).

L'importance et la diffusion croissantes de ces pratiques dans des sociétés occidentales de plus en plus mondialisées s'accompagnent de nouvelles exigences théoriques et méthodologiques. Toutefois, selon Coleman, «nous ne devrions pas affirmer trop vite que le rituel fait brusquement face à de nouveaux défis dans le contexte de la modernité avancée : cela reviendrait à rétablir une distinction obsolète entre sociétés de tradition et sociétés de changement »(2013).

Talal Asad (1993) et Catherine Bell $(1993,1997)$ ont ainsi montré que non seulement les rituels, mais également les théories du rituel sont rigoureusement liés aux processus historiques dans lesquels ils s'inscrivent. Les pratiquants n'ont-ils commencé que depuis peu à se réapproprier, en les modifiant et en les recréant, des rituels qui auraient été plus ou moins immuables dans le 
passé ? Rien ne permet de l'affirmer. De fait, les études historiques et anthropologiques sur le catholicisme et le christianisme vernaculaires ${ }^{6}$ suggèrent que, à toute époque, la religion et le rituel, en tant qu'expérience vécue, sont des phénomènes hétérogènes et fluctuants ${ }^{7}$. La nouveauté, semble-t-il, c'est la mesure dans laquelle cette créativité est autorisée, assumée et encouragée dans la spiritualité contemporaine.

Cette créativité rituelle, qui mobilise aussi bien des connaissances scientifiques qu'ésotériques, constitue un trait important du type de spiritualité qui attire un nombre croissant de personnes en Occident depuis les années 1960. À la différence de certains chercheurs hésitant à se pencher sur les nouvelles expressions de l'action rituelle, je ne crois pas que celle-ci exclue nécessairement l'expérimentation et l'inventivité ; de fait, comme le montrent les analyses historiques du sacrement de l'Eucharistie, nombre de rituels, sinon la plupart d'entre eux, résultent, dans leur forme actuelle, d'un long processus d'expérimentation (Bell 1989). Pour comprendre un rite ou une religion, il faut pouvoir appréhender les conditions historiques et sociales de sa création. Ce qui rend les formes contemporaines particulièrement intéressantes, c'est qu'elles permettent d'observer un tel processus en train de se faire, et de questionner les personnes qui ont consciemment entrepris cette démarche. $\mathrm{Au}$ lieu de ne voir dans ce type de créativité religieuse et rituelle qu'une forme de plus de réenchantement romantique du monde, ou de lui reprocher de rompre avec la tradition, le chercheur peut, en l'étudiant, apprendre comment aborder les rituels qui apparaissent en Occident dans des sociétés plurielles et diversifiées, instables et soumises à des influences séculières.

Les pèlerines de Dana, mais aussi d'autres pratiquantes spirituelles rencontrées sur le terrain, reconnaissent volontiers qu'elles ont créé leurs rituels en mélangeant des éléments appartenant à plusieurs traditions. Ce qui ne compromet en rien, estiment-elles, ni le potentiel du rituel ni son efficacité. En créant des rituels qui sont neufs tout en se rattachant à une tradition, les pèlerines doivent relever le même défi que dans les autres phénomènes religieux "nouveaux », passés ou présents (voir par exemple Aubin-Boltanski, ce numéro). Pour elles, leurs cérémonies ont été créées en recevant l'inspiration du passé, dans le but explicite d'obtenir une structure rituelle qui, sans être identique, est toutefois « équivalente » aux rites antérieurs, préchrétiens en l'occurrence.

Les rituels auxquels j'ai pris part en Espagne, en Italie et au Portugal ont été créés par et pour des personnes qui sont extrêmement critiques envers les religions institutionnalisées (established), en particulier le catholicisme. Ces pratiquantes spirituelles craignent souvent de se retrouver à nouveau dans des structures rigides et contraignantes - bref, « religieuses ». Ayant lutté pour se libérer du système de croyances de leurs parents (Fedele 2013a : 123-144), elles ne veulent ni céder le contrôle

\footnotetext{
${ }^{6}$ Entre autres, Christian 1972, 2011 ; Bynum 1987, 1991 ; Badone 1990.

${ }^{7}$ Sur le vécu religieux contemporain (ou lived religion) voir Mc Guire 2008.
} 
à une autorité extérieure ni avoir l'impression d'adhérer aveuglément à de nouveaux dogmes. Le recours à une terminologie scientifique donne le sentiment aux membres du Bois de la Déesse que leur rituel et leur croyance en ses effets bénéfiques ne se réduisent pas à un « saut dans la foi ». Toutefois, comme l'a montré l'ethnographie du rituel, l'explication « scientifique » par l'ADN a un effet ambigu. Si d'un côté, grâce à elle, les participantes peuvent donner un sens au rite, de l'autre elles peuvent également, à cause d'elle, se sentir «trop dans leur tête »; aussi optent-elles généralement pour une traduction plus symbolique ou psychologique de la référence scientifique, qui ne les empêche pas d'en ressentir les effets bénéfiques (Houseman 2007, 2010). Ces allers-retours incessants entre la science, la psychologie et la religion leur permettent d'optimiser à la fois la compréhension rationnelle du rituel et la perception de ses effets.

Il est explicitement conseillé aux pratiquants spirituels, dans les livres et les ateliers, mais aussi au cours des rituels eux-mêmes, de ne rien croire a priori et de n'accepter une théorie que si elle leur convient (Fedele et Knibbe 2013; Bowman et Sutcliffe 2000; Pike 2001). Mes interlocutrices connaissaient aussi des moments de partage plus ou moins formalisés pendant lesquels elles pouvaient parler de leurs expériences spirituelles, mais aussi résoudre des problèmes ou des doutes, et même formuler des critiques. Aussi nous donnent-elles un accès privilégié aux incertitudes inhérentes à l'expérience rituelle.

Pour les membres du Bois de la Déesse, de même que dans les groupes religieux du Limburg (Pays-Bas) étudiés par Kim Knibbe dans ce numéro, la certitude est une chose dangereuse, évoquant la manipulation mentale, la perte de contrôle - bref, la domination religieuse, voire l'intégrisme. Si bien que l'incertitude n'est pas perçue négativement comme un manque de foi, mais plutôt comme un état d'esprit à cultiver. Cette idée selon laquelle la certitude religieuse est potentiellement dangereuse semble directement liée aux processus de sécularisation et à une conception qui oppose la religion et la foi à la science et à la rationalité. Dans ce contexte particulier, l'incertitude et notre capacité à douter et à expérimenter deviennent des outils efficaces pour maîtriser le risque d'endoctrinement religieux et la perte de son libre arbitre.

Toutefois, nous l'avons vu ci-dessus, cette incertitude religieuse présente aussi des inconvénients pour les pèlerines. Mes interlocutrices me reprochaient souvent d'être « trop dans ma tête » (Fedele 2013b) et critiquaient les scientifiques, qui rejettent la spiritualité, utilisant uniquement leur cerveau gauche, rationnel et masculin, au détriment de l'hémisphère droit, intuitif et féminin. L'objectif explicite des théoriciens et des pratiquants de la spiritualité contemporaine est d'offrir une alternative au désenchantement du monde dont la science, le rationalisme et la sécularisation sont responsables (Fedele et Knibbe 2013). Ainsi, comme le soutient Peter van der Veer, le spirituel et le 
profane « constituent deux alternatives à la religion institutionnalisée de la modernité euroaméricaine, qui, produites simultanément, sont interconnectées » (2009).

Pour ces pratiquantes spirituelles, la religion, la science et la laïcité sont des alliées dangereuses, qu'elles critiquent et combattent, mais dont elles ne peuvent se passer. En raison même de ses liens avec elles, la certitude leur apparaît risquée, mais aussi désirable par moment. En particulier avant le début d'un rituel, les participantes sont souvent invitées à « cesser d'être dans leur tête » et à se laisser aller à " ressentir » et à "s'abandonner au pouvoir de la cérémonie ». Elles doivent se livrer au rituel en croyant à sa réussite, en sachant qu'elles seront libres après son exécution de dire s'il a réellement fonctionné et de le rejeter le cas échéant. Il semble nécessaire en effet, pour que ça marche, de suspendre le doute le temps du rituel. Maria Antònia n'a ainsi pu profiter du rituel qu'après avoir écarté ses pensées inquiètes; Felicia, quant à elle, distraite par des problèmes secondaires n'a pas réussi à s'y engager. Même si, dans ce contexte, le doute semble un puissant allié contre la « religion », pendant le rituel il devient un compagnon fort encombrant.

Dans sa recherche sur le pèlerinage de Walsingham en Angleterre, Simon Coleman soutient que le rituel peut être perçu comme « générateur de risque » et susciter des sentiments d'incertitude et d'incompétence, contrairement à ce qu'affirment les théories anthropologiques classiques qui en font le « moyen d'atténuer le stress lié au risque et à l'incertitude » (2009: 44). Les réactions des pèlerines de la Sainte-Baume en témoignent, « une prise de distance ou une réconciliation temporaire avec une forme rituelle s'accompagne de constantes négociations et d'un recouvrement de la capacité d'action individuelle par l'intermédiaire du corps ou d'une certaine vision des choses» (Coleman 2009 : 48), mais aussi de l'ironie et du jeu (Coleman et Elsner 1998)

Pour mes interlocutrices en Espagne, en Italie et au Portugal, la pratique rituelle suppose un va-et-vient constant entre incertitude et certitude. Si la certitude est un facteur de risque « religieux », l'incertitude comporte le risque d'un échec rituel. En se fondant sur Knibbe (citant Jackson 1998, ce numéro), il paraît également important pour ces pratiquantes d'établir entre les deux une limite qui les aide à savoir quand suspendre le doute, afin de s'immerger dans l'expérience rituelle en étant capable d'en jouir pleinement.

En même temps, cette mise en suspens du doute nécessaire à l'accomplissement du rite constitue une sorte de «point vide » (Agnès Clerc-Renaud citant Severi 2007, ce numéro), qui permet ensuite aux participantes d'expliquer le cas échéant leur incertitude ou leur impression d'échec. Si, après le rituel, certaines d'entre elles estiment (comme Felicia) que celui-ci n'a pas bien fonctionné, il est alors possible d'attribuer ce sentiment non pas au rituel, qui serait inefficace, mais à leur propre incapacité de suspendre le doute et/ou à l'incapacité du leader de favoriser chez elles cette suspension. 
Le sens construit par le rituel peut être morcelé et variable (Humphrey et Laidlaw 1994). C'est particulièrement vrai pour les pratiquantes que j'ai rencontrées, qui, ayant grandi dans des pays de tradition catholique comme le Portugal, l'Espagne ou l'Italie, ont dû lutter pour s'affranchir des limites que leur imposait, selon elles, leur milieu d'origine. L'analyse des récits de pèlerines espagnoles adhérant au mouvement de la spiritualité de la Déesse montre comment celles-ci testent l'efficacité d'un rituel menstruel et surmontent le sentiment d'incompétence qu'il suscite, tout en négociant ses significations et ses effets.

Dans la spiritualité contemporaine, où l'expérience l'emporte sur la croyance, les personnes qui participent aux nouveaux rituels abordent sans gêne les sentiments d'incertitude ou d'incompétence qu'il leur arrive d'éprouver. Si mes interlocutrices admettent aussi facilement leurs doutes ou leur incapacité à participer, c'est que leur leader spirituel les incite à ne rien croire a priori et à toujours tester une théorie ou une pratique avant de l'adopter. Pour ces pratiquantes, qui entretiennent des relations ambiguës avec la religion, mais aussi la science et la sécularisation, la certitude est potentiellement dangereuse certes, mais elle est aussi un gage d'efficacité rituelle. Formidable levier contre la vérité monolithique imposée par une religion ou une science dominée par les hommes, le doute devient aussi un obstacle à cet abandon confiant et irréfléchi nécessaire au rituel. Les célébrantes sont par conséquent obligées de se livrer à une danse rituelle compliquée, avec en alternance l'incertitude puis la certitude pour partenaires.

Or, loin de se limiter aux nouveaux rituels de la spiritualité contemporaine, le sentiment d'incertitude éprouvé par certains croyants est un aspect propre aux rituels élaborés dans un contexte occidental, pluriel et de plus en plus sécularisé, qui a été peu étudié jusqu'à présent par les sciences sociales. Il serait intéressant d'examiner le rôle de l'incertitude dans d'autres rituels contemporains, tels que les rites de passage créés pour célébrer autrement certaines étapes de la vie (par exemple, Grimes 2000) ou donner un sens à des événements dont les grandes religions se désintéressent (Fellous 2001), comme la fausse couche, la ménopause (Fedele 2013) ou la mort d'un animal de compagnie (Brandes 2009).

Enfin, l'analyse des stratégies rituelles mises en œuvre pour trouver un équilibre entre science et ésotérisme, raison et foi, doute et certitude, devrait permettre de mieux comprendre les efforts déployés par l'acteur rituel pour s'engager - corps et âme pour ainsi dire - dans une pratique spirituelle, tout en continuant à se sentir un esprit libre dans la société plurielle, contemporaine et moderne. 
Asad, Talal. 1993. Genealogies of Religion: Discipline and Power in Christianity and Islam. Baltimore: Johns Hopkins University Press.

Aune, Michael B. and De Marinis, Valerie, eds. 1996. Religious and Social Ritual: Interdisciplinary Explorations. Albany: State University of New York Press.

Badone, Ellen. ed. 1990. Religious Orthodoxy and Popular Faith in European Society. Princeton: Princeton University Press.

Bell, Catherine. 1989. "Ritual, Change and Changing Rituals." Worship 63 (1): 31-41.

Bell, Catherine. 1992. Ritual Theory, Ritual Practice. New York: Oxford University Press

Bell, Catherine. 1997. Ritual. Perspectives and Dimensions. New York: Oxford

Bowman, Marion and Sutcliffe, Steven J. eds. 2000. Beyond New Age. Exploring Alternative Spirituality. Edinburgh: Edinburgh University Press.

Brandes, Stanley 2009 „The Meaning of American Pet Cemetery Gravestones“ Ethnology 48:99-118.

Bynum, Caroline Walker. 1987. Holy Feast, Holy Fast. The Religious Significance of Food to Medieval Women. Berkeley: University of California Press.

- 1991. Fragmentation and Redemption. Essays on Gender and the Human Body in Medieval Religion. New York: Zone Books.

Christian, William A. Jr. 1989 [1972]. Person and God in a Spanish Valley. Rev. ed. Princeton, NJ: Princeton University Press.

Christian, William A. Jr. 2011. Divine Presence in Spain and Western Europe 1500-1960. Visions, Religious Images and Photographs. Budapest: Central European Press.

Claverie, Élisabeth. 2003. Les guerres de la Vierge. Une anthropologie des apparitions. Paris: Gallimard.

Coleman, Simon 2013 "Ritual Remains: Studying Contemporary Pilgrimage," in: A Companion to the Anthropology of Religion. J. Boddy and M. Lambek eds., London: Wiley Blackwell, 294-308.

Coleman, Simon. 2009. "On Mirrors, Masks and Traps: Ambiguity, Risk and 'Lateral Participation' in Ritual.” Journal of Ritual Studies 23 (2): 43-51.

Coleman, Simon and Elsner, J. 1998. "Performing Pilgrimage: Walsingham and the Ritual Construction of Irony," in: Ritual, Performance, Media. F. Hughes-Freeland ed., 46-65. New York: Routledge.

Dianteill, Erwan et al. 2004 La modernité rituelle. Rites politiques et religieux des sociétés modernes. Paris: L'Harmattan

Eller, Cynthia. 1993. Living in the Lap of the Goddess: The Feminist Spirituality Movement in America. Boston: Beacon Press.

Eller, Cynthia. 2000 The Myth of Matriarchal Prehistory. Boston: Beacon Press.

Fedele, Anna. 2013a. Looking for Mary Magdalene. Alternative Pilgrimage and Ritual Creativity at Catholic Shrines in France. New York: Oxford University Press. . 2013b. "2012: The Spiritualized Prophecy That Could not Fail”, Religion and Society: Advances in Research, 4 
2014 “Reversing Eve's Curse. Mary Magdalene Pilgrims and the Creative Ritualization of Menstruation” Journal of Ritual Studies 28.2.

Fedele, Anna and Kim Knibbe, eds. 2013. Gender and Power in Contemporary Spirituality. Ethnographic Approaches. New York: Routledge.

Fellous, Michèle. 2001. A la recherche de nouveaux rites. Paris: L'Harmattan.

Geertz, Clifford. 1973. The Interpretation of Cultures. Basic Books.

Grimes, Ronald. 2000. Deeply Into the Bone; Re-inventing Rites of Passage. Berkeley: University of California Press.

Grimes, Ronald et al. 2011 Ritual, Media, and Conflict. Oxford and New York: Oxford University Press

Handelman, Don and Galina Linquist. 2004. Ritual in Its Own Right. Exploring the Dynamics of Transformation. New York: Berghahn Books.

Houseman, Michael. 2007. "Menstrual Slaps and First Blood Celebrations. Inference, Simulation and the Learning of Ritual," in Learning Religion. Anthropological Approaches. D. Berliner and R. Sarró, eds., 31-48. Oxford: Berghahn Books.

- 2010. "Des rituels contemporains de permière menstruation," in Ethnologie française 40 (1): 57-66.

Humphrey, Caroline and Laidlaw, James. 1994. The Archetypal Actions of Ritual: A Theory of Ritual Illustrated by the Jain Rite of Worship. Oxford: Clarendon Press.

McGuire, Meredith. 2008. Lived Religion. Faith and Practice in Everyday Life. Oxford: Oxford University Press.

Pike, Sarah M. 2001. Earthly Bodies, Magical Selves: Contemporary Pagans and the Search for Community. Berkeley: University of California Press.

2004. New Age and Neopagan Religions in America. New York: Columbia University Press.

Rappaport, Roy. 1999. Ritual and Religion in the Making of Humanity. Cambridge: Cambridge University Press.

Van der Veer, Peter 2009 "Spirituality in Modern Society," Social Research: An International Quarterly 76, no. 4: 1097-1120.

Wood, Matthew. 2007. Possession, Power and the New Age: Ambiguities of Authority in Neoliberal Societies. First. Ashgate. 